Pacific Journal of Mathematics

ON THE SPECTRA OF MULTIPLIERS 


\title{
ON THE SPECTRA OF MULTIPLIERS
}

\author{
Misha ZAFRAN
}

In this note, some results concerning the spectral theory and Banach algebra properties of multipliers on compact Abelian groups are obtained. The study concentrates on multipliers whose spectra are, in a sense, natural, and whose transforms vanish at $\infty$. The results are shown to be of particular interest in the case of the measure algebra $M(G)$. Moreover, a necessary and sufficient condition is found for the spectrum of a Riesz product to equal the closure of the range of its Fourier-Stieltjes transform.

Let $G$ be a locally compact Abelian group (or LCA group) with dual group $\Gamma$. For $1 \leqq p \leqq \infty, L_{p}(G)$ will denote the usual $L_{p}$ space with respect to the Haar measure of $G$. Let $M(G)$ designate the space of regular Borel measures on $G$, and let $M_{0}(G)=\left\{\mu \in M(G) \mid \mu^{\wedge}\right.$ vanishes at $\infty\}$, where $\mu^{\wedge}$ denotes the Fourier-Stieltjes transform of $\mu$.

In this note, we will investigate some properties of those multipliers on $L_{p}(G)$ whose spectra are, in a sense, natural. Our major result is Theorem 3.1. We shall see that this theorem is of particular interest for $p=1$. In this case, we are able to obtain new results concerning the spectral theory and Banach algebra properties of certain subalgebras of $M_{0}(G)$-see Theorems $3.2,3.6,3.12,3.13,3.14$, and 3.15. Moreover, using the techniques developed here, we are able to determine the spectra of certain measures in $M_{0}(G)$. In particular, we find a necessary and sufficient condition for the spectrum of a Riesz product to equal the closure of the range of its Fourier-Stieltjes transform-see Theorems 3.6, 3.9, and Remark 3.10.

We begin our discussion with some basic notations used throughout this paper.

1. Notations and definitions. For any Banach space $X$, we let $O(X)$ denote the algebra of bounded linear operators on $X ;\|T\|_{o(X)}$ will denote the norm of an operator $T \in O(X)$. If $x$ is an element of a Banach algebra $A$, we denote the spectrum of $x$ in $A$ by $\operatorname{sp}(x, A)$, and the spectral radius of $x$ by $r_{A}(x) . \quad R(\lambda, x)$ will denote the resolvent of $x$. If $f$ is a function analytic in a neighborhood of $\operatorname{sp}(x, A)$, we let $f(x)$ be the element $(1 / 2 \pi i) \int_{C} f(\lambda) R(\lambda, x) d \lambda$, where $C$ is an envelope of $\mathrm{sp}(x, A)$ contained in the domain of $f$. If $A$ is any commutative Banach algebra, $\Delta(A)$ will designate the maximal ideal space of $A$. If $T \in O(X)$, we denote by $\sigma_{p}(T), \sigma_{r}(T)$, and $\sigma_{c}(T)$ the point, residual, and continuous spectra of $T$, respectively. The symbol $*$ will always 
denote convolution; the symbol $f^{\wedge}$ is the Fourier transform of $f$.

If $A$ and $B$ are any sets, $A \backslash B$ will designate the set theoretic difference between $A$ and $B . A^{c}$ will be the complement of $A$. Finally, if $E$ is any subset of a topological space, $\bar{E}$ will denote the closure of $E$, int $E$ will denote the interior of $E$, and $C(E)$ will denote all continuous, complex-valued functions on $E$.

If $G$ is an LCA group and $1 \leqq p \leqq \infty, M_{p}(G)$ will denote the subalgebra of $O\left(L_{p}(G)\right)$ consisting of those operators which commute with all translations on $L_{p}(G)$. The following properties of $M_{p}(G)$ are well known; see [6].

(1) If $1 \leqq p<\infty$ and $T \in M_{p}(G)$, or if $T \in M_{\infty}(G)$ and $T$ is continuous with respect to the weak* topology of $L_{\infty}(G)$, then there exists a unique function $T^{\wedge} \in L_{\infty}(\Gamma)$ such that $T(f)^{\wedge}=T^{\wedge} f^{\wedge}$ a.e. on $\Gamma$, for all integrable simple functions on $G$. $T^{\wedge}$ will be called the transform of $T$.

We denote by $C M_{p}(G)$ those multipliers $T \in M_{p}(G)$ for which $T^{\wedge}$ is continuous on $\Gamma, 1 \leqq p<\infty$. We write $C_{0} M_{p}(G)=\left\{T \in C M_{p}(G) \mid T^{\wedge}\right.$ vanishes at $\infty$.

(2) If $1 \leqq p<\infty$, then $M_{p}(G)$ is a commutative Banach algebra which is isometric and isomorphic to $M(G)$ if $p=1$, and to $L_{\infty}(\Gamma)$ if $p=2$. Also, $C_{0} M_{p}(G)$ is a Banach algebra.

(3) If $1 \leqq p, q<\infty$ and $|1 / q-1 / 2| \leqq|1 / p-1 / 2|$, then $M_{p}(G) \subseteq$ $M_{q}(G)$, and for all $T \in M_{p}(G),\left\|T^{\wedge}\right\|_{L_{\infty}(T)}=\|T\|_{o\left(L_{2}\right)} \leqq\|T\|_{o\left(L_{q}\right)} \leqq\|T\|_{o\left(L_{p}\right)} \cdot$ Also, if $1<p<\infty$ and $1 / p+1 / p^{\prime}=1$, then $M_{p}(G)$ and $M_{p^{\prime}}(G)$ are isometric and isomorphic.

(4) Let $1 \leqq p<\infty$. Then

(a) $\operatorname{sp}\left(T, O\left(L_{p}\right)\right)=\operatorname{sp}\left(T, M_{p}(G)\right)$, for all $T \in M_{p}(G)$.

(b) $\operatorname{sp}\left(T, O\left(L_{p}\right)\right)=\operatorname{sp}\left(T, C_{0} M_{p}(G)\right)$, for all $T \in C_{0} M_{p}(G)$.

We remark that 4(a) holds since if $S$ is any bounded operator commuting with translations, and if $S^{-1}$ exists, then $S^{-1}$ commutes with translations. Moreover, 4(b) follows from 4(a) as may be seen by adjoining an identity to $C_{0} M_{p}(G)$, and noting that $\left(S^{-1}\right)^{\wedge}=1 / S^{\wedge}$, whenever $S^{-1}$ exists.

2. In this section, we prove a series of lemmas needed in the proof of our basic result. Some of these are quite elementary; others may have certain intrinsic interest.

Before we begin, let us make the following observation. If $f \in L_{1}(G)$, and if we define

$$
T_{f}(g)=f * g
$$

for all $g \in L_{p}(G)$, then $T_{f} \in C_{0} M_{p}(G)$, and $\operatorname{sp}\left(T_{f}, O\left(L_{p}\right)\right)=f^{\wedge}(\Gamma) \cup\{0\}$, $1 \leqq p<\infty$. This is well known and is simple to prove.

However, if $G$ is a nondiscrete LCA group, then $M_{0}(G)$ is not 
a symmetric Banach algebra. This may be seen by combining Theorem $R$ of [9], Corollary 5.6.9(a) of [8], and the argument used in the proof of Theorem 5.3.4 of [8]. It follows that there exists $\mu \in M_{0}(G)$ such that $\operatorname{sp}(\mu, M(G)) \neq \mu^{\wedge}(\Gamma) \cup\{0\}$. Hence by (2) and (4) of the previous section, $\operatorname{sp}\left(T_{\mu}, O\left(L_{1}\right)\right) \neq \mu^{\wedge}(\Gamma) \cup\{0\}$, where $T_{\mu}(g)=\mu * g$ for all $g \in L_{1}(G)$.

Corresponding results seem to be unknown for the Banach algebra $C_{0} M_{p}(G), 1<p<\infty, p \neq 2$. If $p=2$, it is a simple consequence of the Plancherel theorem that $\operatorname{sp}\left(T, O\left(L_{2}\right)\right)=T^{\wedge}(\Gamma) \cup\{0\}$, for all $T \in C_{0} M_{2}(G)$.

We will study those elements $T \in C_{0} M_{p}(G)$ for which $\operatorname{sp}\left(T, O\left(L_{p}\right)\right)=$ $T^{\wedge}(\Gamma) \cup\{0\}$.

If $\varphi$ is an essentially bounded, complex-valued function on a measure space $(\Omega, \Sigma, \mu)$, we define the essential range of $\varphi$ (or ess range $\varphi$ ) as the set of all complex numbers $z$ such that

$$
\mu\{\omega \in \Omega|| f(\omega)-z \mid<\varepsilon\}>0
$$

for every $\varepsilon>0$.

LemMA 2.1. Let $G$ be an LCA group, let $1 \leqq p<\infty$, and let $T \in M_{p}(G)$. Then ess range $T^{\wedge} \subseteq \operatorname{sp}\left(T, O\left(L_{p}\right)\right)$.

Proof. Suppose $\lambda \notin \operatorname{sp}\left(T, O\left(L_{p}\right)\right)$. Then since $\operatorname{sp}\left(T, O\left(L_{p}\right)\right)=$ $\operatorname{sp}\left(T, M_{p}(G)\right)$, we see $S=R(\lambda, T) \in M_{p}(G)$. We then note $I=$ $S \circ(\lambda I-T)$, and hence

$$
1=S^{\wedge}\left(\lambda-T^{\wedge}\right) \text { a.e. on } \Gamma \text {, }
$$

where $S^{\wedge}$ is the transform of $S$. But $S^{\wedge} \in L_{\infty}(\Gamma)$, and so by (1), $\lambda \notin$ ess range $T^{\wedge}$. This concludes the proof.

We remark that if $T \in C_{0} M_{p}(G)$, then ess range $T^{\wedge}=\overline{T^{\wedge}(\Gamma)}=$ $T^{\wedge}(\Gamma) \cup\{0\}$.

Lemma 2.2. Let $1 \leqq p<\infty$, and let $T \in C M_{p}(G)$. If $\lambda$ is an isolated point of $\operatorname{sp}\left(T, O\left(L_{p}\right)\right)$, then $\lambda \in T^{\wedge}(\Gamma)$. Moreover, $\lambda$ is a simple pole of the vector-valued analytic function $R(z, T)$.

Proof. Choose $\varepsilon>0$ such that if $D=\{z|| z-\lambda \mid<\varepsilon\}$, then $\bar{D} \cap \operatorname{sp}\left(T, O\left(L_{p}\right)\right)=\{\lambda\}$. We expand $R(z, T)$ in a Laurent series about $\lambda$ :

$$
R(z, T)=\sum_{n=-\infty}^{\infty} A_{n}(z-\lambda)^{n} \text { for }|z-\lambda|<\varepsilon,
$$

where $A_{n}=(2 \pi i)^{-1} \int_{\varphi} R(z, T)(z-\lambda)^{-n-1} d z$ for all integers $n$, and $\varphi$ denotes the curve $|z-\lambda|=\varepsilon$.

Let $f$ be a function analytic in a neighborhood of $\operatorname{sp}\left(T, O\left(L_{p}\right)\right)$ for 
which $f=1$ on $\bar{D}$ and $f=0$ on $\operatorname{sp}\left(T, O\left(L_{p}\right)\right) \backslash\{\lambda\}$. Then for $n \leqq-1$,

$$
A_{n}=\frac{1}{2 \pi i} \int_{C} f(z)(z-\lambda)^{-n-1} R(z, T) d z,
$$

where $C$ is an envelope of $\operatorname{sp}\left(T, O\left(L_{p}\right)\right)$ contained in the domain of $f$. Hence we see

$$
A_{n}=f(T)(T-\lambda I)^{-n-1} \text { for } n \leqq-1 .
$$

By (4) of $\S 1$, it is evident that both $A_{n}$ and $f(T)$ are in $C M_{p}(G)$ and

$$
f(T)^{\wedge}=f \circ T^{\wedge} \quad \text { on } \quad \Gamma \text {. }
$$

Since $R(z, T)$ is not analytic at $\lambda$, there exists a positive integer $m$ for which $A_{-m} \neq 0$. Therefore, by $(1), f(T) \neq 0$. Hence by (2), there exists $\gamma \in \Gamma$ such that

$$
f\left(T^{\wedge}(\gamma)\right) \neq 0 .
$$

Recalling that ess range $T^{\wedge} \subseteq \mathrm{sp}\left(T, O\left(L_{p}\right)\right)$, an application of (3) and the definition of $f$ yield that $T^{\wedge}(\gamma)=\lambda$. This implies the first assertion of our theorem.

In order to obtain the second assertion, we suppose, to the contrary, that there exists a positive integer $j \geqq 2$ for which $A_{-j} \neq 0$.

Hence there exists $\gamma_{1} \in \Gamma$ for which $\left(A_{-j}\right)^{\wedge}\left(\gamma_{1}\right) \neq 0$. By (1) and (2),

$$
0 \neq\left(A_{-j}\right)^{\wedge}\left(\gamma_{1}\right)=f\left(T^{\wedge}\left(\gamma_{1}\right)\right)\left(T^{\wedge}\left(\gamma_{1}\right)-\lambda\right)^{j-1} .
$$

Thus by (4), $f\left(T^{\wedge}\left(\gamma_{1}\right)\right) \neq 0$ so again we see $T^{\wedge}\left(\gamma_{1}\right)=\lambda$. But since $j \geqq 2$, this contradicts (4). This concludes the proof of Lemma 2.2.

The following theorem will not be needed in the sequel; however, it may be of some interest.

Theorem 2.3. Let $1 \leqq p<\infty$, and let $T \in C M_{p}(G)$. If $\lambda$ is an isolated point of $\mathrm{sp}\left(T, O\left(L_{p}\right)\right)$, then $\lambda \in$ the point spectrum of $T$.

Proof. By Lemma 2.2, we see $\lambda$ is also an isolated point of $\overline{T^{\wedge}(\Gamma)}$. Let $V$ be an open subset of $\Gamma$ such that $\bar{V}$ is compact and $\overline{T^{\wedge}(V)}=\{\lambda\}$. Let $U$ be an open subset of $\Gamma$, with compact closure, such that $U \subseteq \bar{U} \subseteq V$. By [8], Theorem 2.6.2, there exists $f \in L_{1}(G)$ with $f^{\wedge}=1$ on $U$ and $f^{\wedge}=0$ on $V^{c}$.

Since $\bar{V}$ is compact, $f^{\wedge} \in L_{1}(\Gamma)$. Hence by the inversion theorem,

$$
f(x)=\int_{\Gamma} f^{\wedge}(\gamma) \gamma(x) d \gamma
$$

for almost all $x \in G$. In particular, $f \in L_{\infty}(G)$. Thus, since $f$ is also 
in $L_{1}(G)$, we see $f \in L_{p}(G)$.

Finally we note $(\lambda I-T)(f)^{\wedge}=0$ on $\Gamma$. This equality follows since $T^{\wedge}(V)=\{\lambda\}$ and by the choice of $f$. Hence $(\lambda I-T)(f)=0$, and $f \neq 0$. Our assertion follows immediately.

Before proceeding, we make the following observations: If $1 \leqq p \leqq 2$, and if $T \in M_{p}(G)$, then the point spectrum of $T$ is contained in $T^{\wedge}(\Gamma)$. Secondly, we remark that for $1 \leqq p<\infty$ and for compact $G$, we actually obtain $T^{\wedge}(\Gamma)=\sigma_{p}(T)$, since for all

$$
\gamma \in \Gamma,\left(T^{\wedge}(\gamma) I-T\right)(\gamma)=0
$$

Lemma 2.4. Let $G$ be a compact LCA group. Let $1 \leqq p<\infty$, and let $T \in M_{p}(G)$. Then $\sigma_{r}(T)=\varnothing$.

Proof. Let $\lambda \in \operatorname{sp}\left(T, O\left(L_{p}\right)\right)$, with $\lambda \notin \sigma_{p}(T)$. It is then simple to verify that range $(\lambda I-T)$ contains the class $\mathscr{P}$ of all trigonometric polynomials on $G$. Since $G$ is compact, $\mathscr{P}$ is dense in $L_{p}(G)$. Hence range $(\lambda I-T)$ is dense in $L_{p}(G)$, and so $\lambda \in \sigma_{c}(T)$. Our conclusion is immediate.

Combining Lemma 2.4 and the comments preceding it, we obtain:

LEMMA 2.5. Let $G$ be a compact $L C A$ group, and let $1 \leqq p<\infty$. Let $T \in C_{0} M_{p}(G)$. Then $\sigma_{c}(T)$ contains a nonzero point if and only if $\operatorname{sp}\left(T, O\left(L_{p}\right)\right) \neq T^{\wedge}(\Gamma) \cup\{0\}$.

We note that if $G$ is compact, $\Gamma$ is discrete. Hence if $T \in C_{0} M_{p}(G), T^{\wedge}(\Gamma)$ is countable. We will need the following lemma.

Lemma 2.6. Let $G$ be a compact $L C A$ group, and let $1 \leqq p<\infty$. Let $T \in C_{0} M_{p}(G)$. Suppose $\operatorname{sp}\left(T, O\left(L_{p}\right)\right) \neq T^{\wedge}(\Gamma) \cup\{0\}$. Then $\overline{\sigma_{c}(T) \backslash\{0\}}$ is a nonempty perfect set; in particular, $\sigma_{c}(T) \backslash\{0\}$ is uncountable.

Proof. By Lemma 2.5, $\sigma_{c}(T) \backslash\{0\}$ is nonempty. Let $z \in \sigma_{c}(T) \backslash\{0\}$. By the remarks preceding Lemma 2.4, and the lemma itself, $\operatorname{sp}\left(T, O\left(L_{p}\right)\right)$ is the union of the disjoint sets $T^{\wedge}(\Gamma) \cup\{0\}$ and $\sigma_{c}(T) \backslash\{0\}$. Thus $z \notin T^{\wedge}(\Gamma) \cup\{0\}$.

By Lemma 2.2, $z$ is not an isolated point of $\operatorname{sp}\left(T, O\left(L_{p}\right)\right)$. Hence there exists a sequence $\left\{z_{n}\right\}$ of distinct points in $\operatorname{sp}\left(T, O\left(L_{p}\right)\right)$ such that $z_{n} \rightarrow z$ as $n \rightarrow \infty$.

Since $T^{\wedge}(\Gamma) \cup\{0\}$ is compact and $z \notin T^{\wedge}(\Gamma) \cup\{0\}$, we see that at most finitely many of the points $z_{n}$ can be in $T^{\wedge}(\Gamma) \cup\{0\}$. We may thus assume $z_{n} \in \sigma_{c}(T) \backslash\{0\}$ for all $n$.

We have shown that every point of the nonempty set $\sigma_{c}(T) \backslash\{0\}$ is a limit point of the set $\sigma_{c}(T) \backslash\{0\}$. Thus $\overline{\sigma_{c}(T) \backslash\{0\}}$ is a perfect set. From this, it follows easily that $\sigma_{c}(T) \backslash\{0\}$ is uncountable, since 
sp $\left(T, O\left(L_{p}\right)\right)$ is the union of $\sigma_{c}(T) \backslash\{0\}$ and the countable set $T^{\wedge}(\Gamma) \cup\{0\}$. The proof of the lemma is complete.

We will also require the following two lemmas.

Lemma 2.7. Let $G$ be a compact LCA group, and let $1 \leqq p<\infty$. Let $S, T \in C_{0} M_{p}(G)$, and suppose $\quad \operatorname{sp}\left(S, O\left(L_{p}\right)\right)=S^{\wedge}(\Gamma) \cup\{0\}$ and $\operatorname{sp}\left(T, O\left(L_{p}\right)\right)=T^{\wedge}(\Gamma) \cup\{0\} . \quad$ Then $\operatorname{sp}\left(S+T, O\left(L_{p}\right)\right)=(S+T)^{\wedge}(\Gamma) \cup\{0\}$.

Proof. By $4\left(\right.$ b) of $\S 1$, we note $\operatorname{sp}\left(S+T, O\left(L_{p}\right)\right)=\operatorname{sp}\left(S+T, C_{0} M_{p}(G)\right)$. Hence, by the Gelfand theory,

$$
\begin{aligned}
& \operatorname{sp}\left(S+T, O\left(L_{p}\right)\right)=\left\{h(S+T) \mid h \in \Delta C_{0} M_{p}(G)\right\} \cup\{0\} \\
\subseteq & {\left[\left\{h(S) \mid h \in \Delta \mathrm{C}_{0} M_{p}(G)\right\} \cup\{0\}\right]+\left[\left\{h(T) \mid h \in \Delta \mathrm{C}_{0} M_{p}(G)\right\} \cup\{0\}\right] } \\
= & \operatorname{sp}\left(S, O\left(L_{p}\right)\right)+\operatorname{sp}\left(T, O\left(L_{p}\right)\right),
\end{aligned}
$$

where for any subsets $A$ and $B$ of the complex numbers, $A+B=$ $\{a+b \mid a \in A, b \in B\}$.

However, by hypothesis, both $\operatorname{sp}\left(S, O\left(L_{p}\right)\right)$ and $\operatorname{sp}\left(T, O\left(L_{p}\right)\right)$ are countable sets. Thus by $(1)$, sp $\left(S+T, O\left(L_{p}\right)\right)$ is countable. By Lemma 2.6, $\operatorname{sp}\left(S+T, O\left(L_{p}\right)\right)=(S+T)^{\wedge}(\Gamma) \cup\{0\}$. This completes the proof.

Lemma 2.8. Let $G$ be a compact $L C A$ group, and let $1 \leqq p<\infty$. Let $m_{p}(G)$ denote the closure of $\left\{T_{f} \mid f \in L_{1}(G)\right\}$ in the norm of $O\left(L_{p}\right)$ (see the beginning of $\S 2$ for notations). Let $\mathscr{S}$ be any closed subalgebra of $C_{0} M_{p}(G)$ containing $m_{p}(G)$. Suppose $h \in \Delta \mathscr{S} \backslash \Gamma$. Then $h(T)=0$ for all $T \in m_{p}(G)$.

Proof. Assume, to the contrary, that $h(T) \neq 0$. Then $h \in \Delta m_{p}(G)$. However, it is easy to see that $\Delta m_{p}(G)=\Gamma$. Thus there exists $\gamma \in \Gamma$ such that

$$
h(U)=U^{\wedge}(\gamma)
$$

for all $U \in m_{p}(G)$.

We show that (1) is actually valid for all $U \in \mathscr{S}$. Let $f$ be a trigonometric polynomial on $G$ with $f^{\wedge}(\gamma)=1$. Then for all $U \in \mathscr{S}$, we have by (1) that

$$
\begin{gathered}
h(U)=h(U) f^{\wedge}(\gamma)=h(U) h\left(T_{f}\right) \\
=h\left(U \circ T_{f}\right)=U \circ T_{f}^{\wedge}(\gamma)=U^{\wedge}(\gamma) .
\end{gathered}
$$

But $h \in \Delta \mathscr{S} \mid \Gamma$. This contradiction proves the lemma.

We conclude this section with some comments concerning the algebra $m_{p}(G)$, defined in the statement of 2.8. Let $G$ be any LCA group, and let $1 \leqq p<\infty$. We define $m_{p}(G)$ as the closure of $\left\{T_{f} \mid f \in L_{1}(G)\right\}$ in $O\left(L_{p}\right)$. Then $m_{p}(G)$ becomes a Banach algebra with 
maximal ideal space $\Gamma$. (See [5], Theorem 1.17.) Figà-Talamanca and Gaudry [3] have shown that if $G$ is the $n$-torus or Euclidean $n$-space, then $m_{p}(G)$ is a proper subalgebra of $C_{0} M_{p}(G)$ for $1<p<\infty$, $p \neq 2$. In fact, more is true. We have the following:

Proposition 2.9. Let $1 \leqq p<\infty$ with $p \neq 2$. Then, in general, $m_{p}(G)$ is a proper subset of $\mathscr{C}=\left\{T \in C_{0} M_{p}(G) \mid \operatorname{sp}\left(T, O\left(L_{p}\right)\right)=\right.$ $\left.T^{\wedge}(\Gamma) \cup\{0\}\right\}$

Proof. We note first that $m_{p}(G) \subseteq \mathscr{C}$ by Lemma $2.8,1 \leqq p<\infty$. If $p=1$, we let $G$ be any nondiscrete LCA group. In [4], Theorem 5.6, an example is given of a singular measure $\mu \in M_{0}(G)$ such that $\mu * \mu=\mu^{2} \in L_{1}(G)$. Using an argument as in 2.8 , it is simple to verify that $\operatorname{sp}(\mu, M(G))=\mu^{\wedge}(\Gamma) \cup\{0\}$. Defining the operator $T_{\mu}$ by $T_{\mu}(f)=$ $\mu * f$ for all $f \in L_{1}(G)$, we see $T_{\mu} \notin m_{1}(G)$, but $T_{\mu} \in \mathscr{C}$.

Now let $1<p<2$. Let $G$ be the circle group. We choose $T$ as the multiplier of Figà-Talamanca and Gaudry corresponding to $p$, as defined in [3]. Then $T \notin m_{p}(G)$. Moreover, if $r=2 p / 2-p, T^{\wedge}(m)=$ $\pm 1 / 2^{n / r}$, for $2^{n} \leqq m \leqq 2^{n+1}-1, n=0,1,2 \cdots$, and $T^{\wedge}(m)=0$ for $m \leqq 0$. Hence $T^{2 \wedge}$ is of uniformly bounded variation on all dyadic "intervals" of integers. By the classical Marcinkiewicz multiplier theorem (see [11], Chapter 15, Theorem 4.14), $T^{2} \in C_{0} M_{q}(G), 1<q<\infty$. Thus by 16.6.2 of [2], $T^{2} \in m_{q}(G), 1<q<\infty$. By Lemma 2.8, $\operatorname{sp}\left(T, O\left(L_{p}\right)\right)=$ $T^{\wedge}(\Gamma) \cup\{0\}$, where $\Gamma=$ the integers.

Finally if $2<p<\infty$, we let $1 / p+1 / p^{\prime}=1$. Again we let $G=$ the circle group. Let $T$ be the multiplier of Figà-Talamanca corresponding to $p^{\prime}$. The above result, combined with a simple duality argument, shows $T \notin m_{p}(G)$ and $\operatorname{sp}\left(T, O\left(L_{p}\right)\right)=T^{\wedge}(\Gamma) \cup\{0\}$. The proof of the proposition is complete.

Of course, if $p=2$, it is clear that $\mathscr{C}=m_{2}(G)$, for any LCA group $G$.

3. In this section we prove our principal theorems, and give some applications. We also give some examples showing that our results cannot be significantly improved. The following theorem is our principal result.

THEOREM 3.1. Let $G$ be a compact LCA group, and let $1 \leqq p<\infty$. Define $\mathscr{C}=\left\{T \in C_{0} M_{p}(G) \mid \operatorname{sp}\left(T, O\left(L_{p}\right)\right)=T^{\wedge}(\Gamma) \cup\{0\}\right\}$.

(a) If $h \in \Delta C_{0} M_{p}(G) \backslash \Gamma$, then $h(T)=0$ for all $T \in \mathscr{C}$.

(b) $\mathscr{C}$ is a closed ideal in $C_{0} M_{p}(G)$.

(c) $\Delta \mathscr{C}=\Gamma$.

Proof. We first prove (a). Let $T \in \mathscr{C}$, and let $h \in \Delta C_{0} M_{p}(G) \backslash \Gamma$. 
Choose $\varepsilon>0$. Then there exists a compact set $K \cong \Gamma$ such that $\left|T^{\wedge}(\gamma)\right|<\varepsilon$ for $\gamma \notin K$. Let $f$ be a trigonometric polynomial such that $f^{\wedge}=1$ on $K$, and $0 \leqq f^{\wedge} \leqq 1$. Define $T_{1}=T \circ T_{f}$ and $T_{2}=T-T \circ T_{f}$. It is clear that $T=T_{1}+T_{2}$. By Lemma 2.8,

$$
\varphi\left(T_{1}\right)=0 \text { for all } \varphi \in \Delta C_{0} M_{p}(G) \backslash \Gamma .
$$

In particular, sp $\left(T_{1}, O\left(L_{p}\right)\right)=T_{1}^{\wedge}(\Gamma) \cup\{0\}$. By Lemma 2.7,

$$
\operatorname{sp}\left(T_{2}, O\left(L_{p}\right)\right)=T_{2}^{\wedge}(\Gamma) \cup\{0\} .
$$

We now show

$$
\left|h\left(T_{2}\right)\right|<\varepsilon .
$$

Note $h\left(T_{2}\right) \in \operatorname{sp}\left(T_{2}, O\left(L_{p}\right)\right)=T_{2}^{\wedge}(\Gamma) \cup\{0\} . \quad$ If $h\left(T_{2}\right)=0$, there is nothing to prove. Otherwise, there exists $\gamma \in \Gamma$ such that $h\left(T_{2}\right)=T_{2}^{\wedge}(\gamma)$. If $\gamma \in K$, the definition of $T_{2}$ implies $T_{2}^{\wedge}(\gamma)=0$. If $\gamma \notin K$,

$$
\left|h\left(T_{2}\right)\right|=\left|T_{2}^{\wedge}(\gamma)\right|=\left|T^{\wedge}(\gamma)\left(1-f^{\wedge}(\gamma)\right)\right|<\varepsilon .
$$

Hence (2) obtains.

Combining (1) and (2) we see

$$
|h(T)|<\varepsilon .
$$

Since $\varepsilon>0$ was arbitrarily chosen, part (a) follows by (3).

Part (b) follows immediately by (a). We now show (c). We begin by noting

$$
\operatorname{sp}(T, \mathscr{C})=\operatorname{sp}\left(T, C_{0} M_{p}(G)\right)
$$

for all $T \in \mathscr{C}$. To see this, we adjoin the identity $I$ to the Banach algebras $\mathscr{C}$ and $C_{0} M_{p}(G)$, and denote the corresponding algebras by $\mathscr{C}_{+}$and $C_{+} M_{p}(G)$ respectively. Then if $T \in \mathscr{C}$, and $\lambda \notin \operatorname{sp}\left(T, C_{0} M_{p}(G)\right)$, $S=(\lambda I-T)^{-1}$ exists and is in $C_{+} M_{p}(G)$. Moreover, $S^{\wedge}$ has limit $1 / \lambda$ at $\infty$. It is now a simple consequence of the spectral mapping theorem that $\operatorname{sp}\left(S-(1 / \lambda) I, O\left(L_{p}\right)\right)=(S-(1 / \lambda) I)^{\wedge}(\Gamma) \cup\{0\}$, and so $S-(1 / \lambda) I \in \mathscr{C}$. Thus $S$ is in the Banach algebra $\mathscr{C}_{+}$. Therefore, $\lambda \notin \operatorname{sp}(T, \mathscr{C})$. We have shown

$$
\operatorname{sp}(T, \mathscr{C}) \subseteq \operatorname{sp}\left(T, C_{0} M_{p}(G)\right)
$$

The reverse inclusion is elementary. Hence (4) is valid.

In order to complete the proof of (c), we assume there exists $h \in \Delta \mathscr{C} \mid \Gamma$. Let $\varepsilon>0$, choose $T \in \mathscr{C}$, and decompose $T$ into the sum $T_{1}+T_{2}$ as in part (a). As before, we have $T_{1}, T_{2} \in \mathscr{C}$. By Lemma 2.8 (with $\mathscr{S}=\mathscr{C}$ ), $h\left(T_{1}\right)=0$. By (4), $h\left(T_{2}\right) \in \operatorname{sp}\left(T_{2}, C_{0} M_{p}(G)\right.$ ). Hence there exists $\gamma \in \Gamma$ such that $h\left(T_{2}\right)=T_{2}^{\wedge}(\gamma)$. The argument of (a) 
implies $|h(T)|<\varepsilon$. Since this holds for all $\varepsilon>0, h(T)=0$. Since $T \in \mathscr{C}$ was arbitrarily chosen, $h=0$ on $\mathscr{C}$, and this contradiction completes the proof of (c). Our theorem is completely proved.

In the case $p=1, C_{0} M_{p}(G)=M_{0}(G)$, and $\mathscr{C}$ is then a proper subalgebra of the asymmetric Banach algebra $M_{0}(G)$. In case $p=2$, $\mathscr{C}=C_{0} M_{p}(G)$, and our theorem becomes trivial. If $1<p<\infty, p \neq 2$, it appears to be unknown whether or not $\mathscr{C}$ is a proper subset of $C_{0} M_{p}(G)$.

In the remainder of this note, we restrict our attention primarily to the case $p=1$, that is, to $M_{0}(G)$ and $M(G)$. Applied to measures, Theorem 3.1 becomes:

THEOREM 3.2. Let $G$ be a compact LCA group. Let

$$
\mathscr{C}=\left\{\mu \in M_{0}(G) \mid \operatorname{sp}(\mu, M(G))=\mu^{\wedge}(\Gamma) \cup\{0\}\right\} .
$$

(a) If $h \in \Delta M_{0}(G) \backslash \Gamma$, then $h(\mu)=0$ for all $\mu \in \mathscr{C}$.

(b) $\mathscr{C}$ is a closed ideal in $M_{0}(G)$.

(c) $\Delta \mathscr{C}=\Gamma$.

We now give some examples to show that 3.2 cannot be significantly strengthened.

EXAMPLE 3.3. We show assertion (a) of 3.2 becomes false if we no longer assume that the measures involved vanish at $\infty$.

Let $x \in G$, and let $\delta_{x}$ be the unit mass measure at $x$. Then $\operatorname{sp}\left(\delta_{x}, M(G)\right)=\{z|| z \mid=1\}$, if the order of $x$ is infinite, and $\mathrm{sp}\left(\delta_{x}, M(G)\right)=$ the $n$th roots of unity, if the order of $x$ is $n$. This is known, and is not difficult to prove. Thus if $h \in \Delta M(G) \backslash \Gamma, h\left(\delta_{x}\right) \in$ $\{z|| z \mid=1\}$. Hence (a) of Theorem 3.2 fails.

ExAmple 3.4. Let $G$ be an $I$-group (see p. 46 of [8]). We show that there exist measures $\mu$ and $\nu$ in $M(G)$ such that

(1) $\operatorname{sp}(\mu, M(G))=\overline{\mu^{\wedge}(\Gamma)}$

(2) $\operatorname{sp}(\nu, M(G))=\overline{\nu^{\wedge}(\Gamma)}$

(3) $\operatorname{sp}(\mu+\nu, M(G))$ properly contains $\overline{(\mu+\nu)^{\wedge}(\Gamma)}$. (Here, neither $\mu^{\wedge}$ nor $\nu^{\wedge}$ vanish at $\infty$ on $\Gamma$.) By Theorem 5.2.2 of [8], there exists a Cantor set $P \leqq G$ which is also a Kronecker set. Let $\mu$ be a positive continuous measure of total variation 1 concentrated on $P$. Then by Theorem 5.5.2 of [8],

$$
\overline{\mu^{\wedge}(\Gamma)}=\{z|| z \mid \leqq 1\} \text {. }
$$

Since $\mu$ has norm 1 , we obtain $\operatorname{sp}(\mu, M(G))=\overline{\mu^{\wedge}(\Gamma)}$. We now define $\nu(E)=\mu(-E)$, where $-E=\{-x \mid x \in E\}$, for all Borel sets $E \leqq G$. The preceding argument also shows sp $(\nu, M(G))=\overline{\nu^{\wedge}(\Gamma)}$. 
However, an examination of the proof of Theorem 5.3.4 of [8] shows that $\overline{(\mu+\nu)^{\wedge}(\Gamma)}$ is a subset of the real numbers, whereas $\mathrm{sp}(\mu+\nu, M(G))$ contains an imaginary number. Thus sp $(\mu+\nu, M(G))$ properly contains $\overline{(\mu+\nu)^{\wedge}(\Gamma)}$.

We will now obtain some consequences of Theorem 3.2. The following result is an immediate corollary of $3.2(\mathrm{c})$.

CoROLlaRy 3.5. Let $G$ be a compact LCA group. Let $\mathscr{C}=$ $\left\{\mu \in M_{0}(G) \mid \operatorname{sp}(\mu, M(G))=\mu^{\wedge}(\Gamma) \cup\{0\}\right\}$. Then the quotient algebra $\mathscr{C} / L_{1}(G)$ has empty maximal ideal space.

This corollary enables us to obtain a new method for showing that a measure in $M_{0}(G)$ does not have a "natural" spectrum. Let $\mu^{1}=\mu$ and $\mu^{n}=\mu^{n-1} * \mu$ for $n \geqq 2$. We have the following result.

THEOREM 3.6. Let $G$ be a compact LCA group, and let $\mu \in M_{0}(G) \backslash\{0\}$. Suppose that for all positive integers $n, \mu^{n}$ is singular with respect to the Haar measure of $G$. Then $\operatorname{sp}(\mu, M(G)) \neq \mu^{\wedge}(\Gamma) \cup\{0\}$.

Proof. Suppose, to the contrary, that $\operatorname{sp}(\mu, M(G))=\mu^{\wedge}(\Gamma) \cup\{0\}$. Then $\mu \in \mathscr{C}$. By the above corollary and the spectral radius formula we obtain

$$
\lim _{n \rightarrow \infty}\left(\left\|\mu^{n}+L_{1}(G)\right\|_{\mathscr{E}\left(L_{1}(G)\right.}\right)^{1 / n}=0 .
$$

Using the definition of the quotient norm we see

$$
\lim _{n \rightarrow \infty}\left(\inf \left\{\left\|\mu^{n}+f\right\| \mid f \in L_{1}(G)\right\}\right)^{1 / n}=0,
$$

where for any measure $\nu,\|\nu\|$ denotes the total variation norm of $\nu$.

Since $\mu^{n}$ is a singular measure, we see $\left\|\mu^{n}+f\right\|=\left\|\mu^{n}\right\|+\|f\| \geqq$ $\left\|\mu^{n}\right\|$ for all $f \in L_{1}(G)$, and for all $n \geqq 1$.

By (1) we thus obtain

$$
\lim _{n \rightarrow \infty}\left\|\mu^{n}\right\|^{1 / n}=0 \text {. }
$$

Hence $\operatorname{sp}(\mu, M(G))=\{0\}$, so by Lemma $2.1, \mu^{\wedge}(\Gamma)=\{0\}$. It follows $\mu=0$, and this contradiction implies the desired result.

EXAMPLE 3.7. Let $G$ be an $I$-group. We show that there exists a continuous measure $\mu \in M(G)$ such that $\mu^{n}$ is singular with respect to the Haar measure of $G$ for all $n \geqq 1$, but such that $\operatorname{sp}(\mu, M(G))=$ $\overline{\mu^{\wedge}(\Gamma)}$. As in Example 3.4, we let $P \subseteq G$ be a Cantor set which is also a Kronecker set, and let $\mu$ be a positive continuous measure concentrated on $P$. By 3.4, $\operatorname{sp}(\mu, M(G))=\overline{\mu^{\wedge}(\Gamma)}$. Let $P_{1}=P$ and $P_{n}=$ 
$P_{n-1}+P$ for $n \geqq 2$. Let $m$ denote the Haar measure of $G$. By [8], Theorem 5.3.6, $m\left(P_{n}\right)=0$ for $n \geqq 1$. But $\mu^{n}$ is concentrated on $P_{n}$, so $\mu^{n}$ is singular with respect to $m$, for all $n \geqq 1$. We note that $\mu^{\wedge}$ does not vanish at $\infty$.

Comment. The above example can be simplified if we no longer require that $\mu$ be a continuous measure. In this case, we let $\nu$ be any discrete measure on $G$. Then clearly $\nu^{n}$ is singular with respect to the Haar measure of $G$ for all $n \geqq 1$. But it is known that $\operatorname{sp}(\nu, M(G))=\overline{\nu^{\wedge}(\Gamma)}$.

REMARK 3.8. Let $G$ be any LCA group. We note that for $\mu \in M_{0}(G), \quad \operatorname{sp}\left(T_{\mu}, O\left(L_{p}\right)\right)=\mu^{\wedge}(\Gamma) \cup\{0\}, \quad$ where $T_{\mu}(f)=\mu * f$ for all $f \in L_{p}(G), 1<p<\infty$. This follows by Theorem 1.16 of [5]. (That theorem is stated for $G=R^{n}$. The proof for arbitrary LCA groups requires no new ideas.)

We will now use Theorem 3.6 to determine the spectrum of a certain class of measures on the circle group.

Let $\left\{a_{k}\right\}$ be a sequence with $a_{k} \neq 0$, and $-1 \leqq a_{k} \leqq 1$ for all $k$. Let $\left\{n_{k}\right\}$ be a sequence of positive integers such that $n_{k+1} / n_{k} \geqq q$ for all $k$, where $q$ is a fixed real number greater than 3 . It is shown in [11], Chapter 5, $\S 7$, that the infinite product

$$
\prod_{k=1}^{\infty}\left(1+a_{k} \cos n_{k} x\right)
$$

represents a positive continuous measure $\mu$ on the circle group called a Riesz product. By the assumption $n_{k+1} / n_{k} \geqq q>3$, it is not difficult to show that

$$
\mu^{\wedge}\left(\sum_{j=1}^{k} \varepsilon_{j} n_{j}\right)=\prod_{j=1}^{k}\left(\frac{\alpha_{j}}{2}\right)^{\left|\varepsilon_{j}\right|}
$$

where $\varepsilon_{j}=0,1$ or -1 for all $j$, and

$$
\mu^{\wedge}(m)=0,
$$

for all $m$ not of the form $\sum_{j=1}^{k} \varepsilon_{j} n_{j}$. (See [11], Chapter 5, $\S 7$, and [10], §2.)

We thus obtain

(a) If $a_{k} \rightarrow 0$ as $k \rightarrow \infty$, then $\mu^{\wedge}$ vanishes at $\infty$.

(b) $\mu^{n}$ is represented by the Riesz product

$$
\prod_{k=1}^{\infty}\left(1+2\left(\frac{a_{k}}{2}\right)^{n} \cos n_{k} x\right)
$$

for all $n \geqq 1$.

In the following theorem only, $G$ will denote the circle group, 
and $\Gamma$ will denote the integers.

THEOREM 3.9 Let $\left\{a_{k}\right\}$ be a sequence of real numbers such that for all $k, a_{k} \neq 0,-1 \leqq a_{k} \leqq 1$, and $a_{k} \rightarrow 0$ as $k \rightarrow \infty$. Let $\left\{n_{k}\right\}$ be $a$ sequence of positive integers with $n_{k+1} / n_{k} \geqq q$, where $q$ is a fixed real number greater than 3. Let $\mu$ be represented by the Riesz product $\prod_{k=1}^{\infty}\left(1+a_{k} \cos n_{k} x\right)$. Then $\operatorname{sp}(\mu, M(G))=\mu^{\wedge}(\Gamma) \cup\{0\}$ if and only if there exists a positive integer $n$ such that $\sum_{k=1}^{\infty}\left|a_{k}\right|^{n}<\infty$.

Proof. We first prove the sufficiency. Choose a positive integer $n$ such that

$$
\sum_{k=1}^{\infty}\left|a_{k}\right|^{n}<\infty \text {. }
$$

By (b) of the preceding paragraph, the measure $\mu^{n}$ is represented by the Riesz product

$$
\prod_{k=1}^{\infty}\left(1+2\left(\frac{a_{k}}{2}\right)^{n} \cos n_{k} x\right)
$$

It is evident by (1) that $\sum_{k=1}^{\infty}\left|2\left(a_{k} / 2\right)^{n}\right|^{2}<\infty$, so by [11], Chapter 5 , Exercise 20, we see $\mu^{n}$ is absolutely continuous with respect to Lebesgue measure, that is $\mu^{n} \in L_{1}(G)$. It follows easily by Lemma 2.8 that $\operatorname{sp}(\mu, M(G))=\mu^{\wedge}(\Gamma) \cup\{0\}$, as desired.

We now prove the necessity of our condition. Suppose for all positive integers $m$ we have

$$
\sum_{k=1}^{\infty}\left|a_{k}\right|^{m}=\infty
$$

Since $a_{k} \rightarrow 0$ as $k \rightarrow \infty$, (a) of the preceding paragraph implies that $\mu \in M_{0}(G)$. Let $n$ be any positive integer. By (b) of the preceding paragraph, $\mu^{n}$ is represented by the Riesz product $\prod_{k=1}^{\infty}\left(1+2\left(a_{k} / 2\right)^{n} \cos n_{k} x\right)$. Moreover, by (2), we see

$$
\sum_{k=1}^{\infty}\left|2\left(\frac{a_{k}}{2}\right)^{n}\right|^{2}=\infty
$$

Hence by [11], Chapter 5, Theorem 7.6, $\mu^{n}$ is singular with respect to Lebesgue measure. By Theorem 3.6, sp $(\mu, M(G)) \neq \mu^{\wedge}(\Gamma) \cup\{0\}$. This concludes the proof.

Comment. A special case of the necessity part of this theorem, with more restrictive assumptions on the sequences $\left\{a_{k}\right\}$ and $\left\{n_{k}\right\}$ is implicit in [10], §2. However, it is not explicitly stated. Our proof is completely different, and much more simple.

REMARK 3.10. The analogue of Theorem 3.9 is valid for more 
general Riesz products on a wider class of compact LCA groups. Explicitly, we let $G$ be one of the three types of groups considered in Theorem 4.4 of [4], and let $\left\{\gamma_{k}\right\}$ be a dissociate set of characters of $G$ as considered in this theorem (for definitions, see [4]). Let $\left\{b_{k}\right\}$ be a sequence of nonzero complex numbers such that $\left|b_{k}\right| \leqq 1 / 2$, if the order of $\gamma_{k}>2$, and such that $b_{k}$ is real with $\left|b_{k}\right| \leqq 1$, if the order of $\gamma_{k}=2$. Let $\mu \in M(G)$ satisfy

$$
\mu^{\wedge}\left(\sum_{j=1}^{k} \varepsilon_{j} \gamma_{j}\right)=\prod_{j=1}^{k} b_{j}{ }^{\left(\varepsilon_{j}\right)},
$$

where $\varepsilon_{j}=0,1$ or -1 for all $j$, and

$$
\mu^{\wedge}(\gamma)=0 \text {, }
$$

for all $\gamma$ not of the form $\sum_{j=1}^{k} \varepsilon_{j} \gamma_{j}$. (See [4], Theorem 3.2.) (Here $b^{(s)}=b$ if $\varepsilon=1, b^{(\varepsilon)}=\bar{b}$ if $\varepsilon=-1$, and $b^{(\varepsilon)}=1$ if $\varepsilon=0$, for all complex numbers $b$.) This may be considered as a generalization of the previously considered Riesz product on the circle group. Suppose also $b_{k} \rightarrow 0$ as $k \rightarrow \infty$. We then have $\operatorname{sp}(\mu, M(G))=\mu^{\wedge}(\Gamma) \cup\{0\}$ if and only if there exists a positive integer $n$ such that $\sum_{k=1}^{\infty}\left|b_{k}\right|^{n}<\infty$. The proof is identical to the one given in 3.9. We need only replace the results of [11], Chapter 5 used in the proof of 3.9 by Theorem 4.4 of [4].

We now give some further applications of Theorem 3.2. We will adhere to the following notation. If $\mu \in M(G)$ and $f \in L_{1}(\mu)$, we define $\nu=f \mu$ as the unique regular Borel measure satisfying $d \nu=f d \mu$. If $\mu, \xi \in M(G)$ with $\mu \geqq 0$, and if $\xi$ is absolutely continuous with respect to $\mu$, we write $\xi \ll \mu$. Finally, if $\mu \in M(G)$, we let $|\mu|$ denote the total variation of $\mu$, and denote by $\|\mu\|$ the total variation norm of $\mu$.

We provide a proof of the following lemma for the convenience of the reader.

Lemma 3.11. Let $G$ be a compact $L C A$ group, and let $\mu \in M_{0}(G)$. If $\nu \in M(G)$, and if $\nu \ll|\mu|$, then $\nu \in M_{0}(G)$.

Proof. Since $\nu$ is absolutely continuous with respect to $|\mu|$, there exists a function $f \in L_{1}(\mu)$ such that $\nu=f \mu$. We show $\nu^{\wedge}$ vanishes at $\infty$. Let $\varepsilon>0$. Then there is a function $g \in C(G)$ such that

$$
\int_{G}|f-g| d|\mu|<\varepsilon / 4
$$

Since $G$ is compact, we can choose a trigonometric polynomial $P=\sum_{k=1}^{n} a_{k} \gamma_{k}$, where $\gamma_{k} \in \Gamma, 1 \leqq k \leqq n$, for which 


$$
\|P-g\|_{\infty}<\frac{\varepsilon}{4(\|\mu\|+1)} .
$$

Combining (1) and (2) we obtain

$$
\int_{G}|f-P| d|\mu|<\varepsilon / 2 \text {. }
$$

Let $\nu_{P}=P \mu$. Then $\nu_{P}{ }^{\wedge}(\gamma)=\sum_{k=1}^{n} a_{k} \mu^{\wedge}\left(\gamma-\gamma_{k}\right)$ for all $\gamma \in \Gamma$. Since $\mu^{\wedge}$ vanishes at $\infty$, there exists a compact set $K \subseteq \Gamma$ such that

$$
\left|\nu_{P}{ }^{\wedge}(\gamma)\right|<\varepsilon / 2
$$

for all $\gamma \notin K$.

Hence by (3) and (4), we see that for $\gamma \notin K$,

$$
\begin{aligned}
\left|\nu^{\wedge}(\gamma)\right| & \leqq\left|\nu^{\wedge}(\gamma)-\nu_{P}{ }^{\wedge}(\gamma)\right|+\left|\nu_{P}{ }^{\wedge}(\gamma)\right| \\
& =\left|\int_{G} \overline{\gamma(x)}(f(x)-P(x)) d \mu(x)\right|+\left|\nu_{P}{ }^{\wedge}(\gamma)\right| \\
& <\varepsilon / 2+\varepsilon / 2=\varepsilon .
\end{aligned}
$$

Thus $\nu^{\wedge}$ vanishes at $\infty$, and our proof is complete.

THEOREM 3.12. Let $G$ be a compact $L C A$ group, and let $\mathscr{C}=$ $\left\{\mu \in M_{0}(G) \mid \operatorname{sp}(\mu, M(G))=\mu^{\wedge}(\Gamma) \cup\{0\}\right\}$. Let $\mu \in \mathscr{C}$. If $\nu \in M(G)$, and if $\nu \ll|\mu|$, then $\nu \in \mathscr{C}$.

Proof. By Lemma 3.11, $\nu \in M_{0}(G)$. We need only show

$$
\operatorname{sp}(\nu, M(G))=\nu^{\wedge}(\Gamma) \cup\{0\} \text {. }
$$

Let $\gamma \in \Gamma$. Then since $\gamma$ is a character of $G$, a simple computation shows that

$$
\left(\gamma \mu_{1}\right) *\left(\gamma \mu_{2}\right)=\gamma\left(\mu_{1} * \mu_{2}\right)
$$

for all $\mu_{1}, \mu_{2} \in M(G)$. In fact, the mapping $\eta \rightarrow \gamma \eta$ for $\eta \in M(G)$ defines an isometric algebra isomorphism of $M(G)$ onto itself.

Define $\mu_{\gamma}=\gamma \mu$, and let $R_{r}(\lambda)=\gamma R(\lambda, \mu)$, for $\lambda \notin \operatorname{sp}(\mu, M(G))$. By (1), it follows that if $\lambda \notin \operatorname{sp}(\mu, M(G)), R\left(\lambda, \mu_{\gamma}\right)$ exists and equals $R_{r}(\lambda)$. Thus $\lambda \notin \operatorname{sp}\left(\mu_{r}, M(G)\right)$ and so $\operatorname{sp}\left(\mu_{r}, M(G)\right) \cong \operatorname{sp}(\mu, M(G))$. Since $\mu=\bar{\gamma} \mu_{\gamma}$, an analogous argument yields $\operatorname{sp}(\mu, M(G)) \subseteq \operatorname{sp}\left(\mu_{\gamma}, M(G)\right)$.

We thus obtain $\operatorname{sp}\left(\mu_{\gamma}, M(G)\right)=\operatorname{sp}(\mu, M(G))=\mu^{\wedge}(\Gamma) \cup\{0\}$. Since $\mu_{\gamma}^{\wedge}(\Gamma)=\mu^{\wedge}(\Gamma)$, it follows

$$
\operatorname{sp}\left(\mu_{\gamma}, M(G)\right)=\mu_{r}{ }^{\wedge}(\Gamma) \cup\{0\} .
$$

Hence $\mu_{r} \in \mathscr{C}$ for all $\gamma \in \Gamma$.

Now let $P=\sum_{k=1}^{n} a_{k} \gamma_{k}$ be a trigonometric polynomial on $G$, and 
let $\nu_{P}=P \mu=\sum_{k=1}^{n} a_{k} \mu_{\gamma_{k}}$. Then since $\nu_{P}$ is a linear combination of elements of $\mathscr{C}$, and since, by Theorem $3.2(\mathrm{~b}), \mathscr{C}$ is a linear space, we obtain

$$
\operatorname{sp}\left(\nu_{P}, M(G)\right)=\nu_{P}{ }^{\wedge}(\Gamma) \cup\{0\} .
$$

Since $\{P \mu \mid P$ is a trigonometric polynomial on $G\}$ is dense in $\left\{f \mu \mid f \in L_{1}(\mu)\right\}$, (2) shows that

$$
\operatorname{sp}(f \mu, M(G))=f \mu^{\wedge}(\Gamma) \cup\{0\},
$$

for all $f \in L_{1}(\mu)$. The theorem now follows by (3), and the fact that $\nu \ll|\mu|$.

By combining Theorems 3.6 and 3.12 , we can obtain an extension of 3.6. For notational convenience, we write $\mu \perp \nu$ if the measures $\mu$ and $\nu$ are mutually singular. If $\mathscr{F}$ is any family of measures, we write $\mu \perp \mathscr{F}$ if $\mu \perp \nu$, for all $\nu$ in $\mathscr{F}$.

THEOREM 3.13. Let $G$ be a compact LCA group with Haar measure m. Define $\mathscr{C}=\left\{\nu \in M_{0}(G) \mid \operatorname{sp}(\nu, M(G))=\nu^{\wedge}(\Gamma) \cup\{0\}\right\}$. Let $\mu \in M(G)$ be a positive measure such that $\mu^{n} \perp m$ for all positive integers $n$. Then $\mu \perp \mathscr{C}$.

Proof. Suppose, to the contrary, that there exists $\nu \in \mathscr{C}$ which is not singular with respect to $\mu$. By Theorem $3.12,|\nu| \in \mathscr{C}$, and it is evident that $|\nu|$ is not singular with respect to $\mu$. We write

$$
|\nu|=\nu_{1}+\nu_{2},
$$

where $\nu_{1}$ and $\nu_{2}$ are positive measures, and

$$
\nu_{1} \ll \mu \text { and } \nu_{2} \perp \mu \text {. }
$$

By the choice of $\nu$, we see $\nu_{1} \neq 0$. Moreover, by (1) and Theorem $3.12, \nu_{1} \in \mathscr{C}$. Now (2) implies that $\nu_{1}^{n} \ll \mu^{n}$ for $n \geqq 1$, and so since $\mu^{n} \perp m$, we see

$$
\nu_{1}^{n} \perp m,
$$

for $n \geqq 1$. But then 3.6 asserts that $\operatorname{sp}\left(\nu_{1}, M(G)\right) \neq \nu_{1}^{\wedge}(\Gamma) \cup\{0\}$, and this contradiction proves the theorem.

We conclude this paper with some results concerning $\mathscr{C}$ as a Banach algebra. Our discussion shows that the behavior of $\mathscr{C}$ is more closely related to that of $L_{1}(G)$ than to that of $M_{0}(G)$.

Let $G$ be a compact, infinite, LCA group, and denote by $B(\Gamma)$ the set $\left\{\mu^{\wedge} \mid \mu \in M(G)\right\}$. Let $A$ be a closed subalgebra of $M(G) . \quad S$ will signify a subset of the complex plane. A complex function $F$ with domain $S$ is said to operate on $A$ if and only if for all $\mu \in A$ with 
$\overline{\mu^{\wedge}(\Gamma)} \cong S$, we have $F\left(\mu^{\wedge}\right) \in B(\Gamma)$. Let $\mathscr{C}^{\wedge}=\left\{\mu^{\wedge} \mid \mu \in \mathscr{C}\right\}$.

A theorem of Katznelson [8], Chapter 6, asserts that if $F$ operates on $L_{1}(G)$, then $F$ is analytic in a neighborhood of the origin. A theorem of Varopoulos [10] asserts that for $G$ compact, only entir functions operate on $B_{0}(\Gamma)$.

We obtain:

THEOREM 3.14. Let $G$ be a compact LCA group.

(a) If $F$ operates on $\mathscr{C}$, then $F$ is analytic in a neighborhood of the origin.

(b) If $F$ is analytic in a neighborhood of 0 , then $F$ operates on C. Moreover, if $F(0)=0$, then $F\left(\mu^{\wedge}\right) \in \mathscr{C}^{\wedge}$ for all $\mu \in \mathscr{C}$ with $\overline{\mu^{\wedge}(\Gamma)} \subseteq$ domain $F$.

This theorem follows by the aforementioned result of Katznelson, Theorem 3.2(c), and the elementary operational calculus of Banach algebras. The details are left to the reader.

Finally, we characterize the homomorphisms of $\mathscr{C}$ into $M(G)$. For all notations used in the following theorem, see [8], Chapter 4.

THEOREM 3.15. Let $G$ be a compact LCA group, and let $\Psi$ be a homomorphism from $\mathscr{C}$ into $M(G)$. Then $\Psi(\mu)^{\wedge}=\mu^{\wedge} \circ \alpha$, where $\alpha$ is a piecewise affine map of $Y$ into $\Gamma$, and $Y$ is in the coset ring of $\Gamma$. Conversely, if $Y$ belongs to the coset ring of $\Gamma$, and if $\alpha$ is a piecewise affine map of $Y$ into $\Gamma$, then $\mu^{\wedge} \circ \alpha \in B(\Gamma)$ for all $\mu \in \mathscr{C}$.

Stated more simply, $\Psi$ is a nontrivial homomorphism of $\mathscr{C}$ into $M(G)$ if and only if $\Psi$ is a nontrivial homomorphism from $L_{1}(G)$ into $M(G)$. This is in sharp contrast to the case of homomorphisms from $M_{0}(G)$ into $M(G)$; there is an example of a nonzero homomorphism $\pi$ from $M_{0}(G)$ into $M(G)$ for which $\left.\pi\right|_{L_{1}(G)}=0$ (see [8], p. 78).

The proof of Theorem 3.15 follows immediately by Theorem 4.1.3 of [8], Theorem 3.2(c), and the argument on p. 77 of [8].

We now have the following corollary.

CoRollary 3.16. Assume the notations of Theorem 3.15. Moreover, suppose that for every finite set $K \subseteq \Gamma, \alpha^{-1}(K)$ is also finite. Then $\Psi$ maps $\mathscr{C}$ into itself.

Proof. We show first that if $\mu \in M_{0}(G)$, then $\Psi(\mu) \in M_{0}(G)$. Let $\varepsilon>0$. Then there exists a finite set $K \cong \Gamma$ such that $\left|\mu^{\wedge}(\gamma)\right|<\varepsilon$ wherever $\gamma \notin K$. Let $J=\alpha^{-1}(K)$. Then $J$ is finite and $\left|\mu^{\wedge}(\alpha(\gamma))\right|<\varepsilon$ whenever $\gamma \notin J$. It follows that $\left|\Psi(\mu)^{\wedge}(\gamma)\right|<\varepsilon$ for $\gamma \notin J$, and so $\Psi(\mu) \in M_{0}(G)$. 
It now suffices to prove

$$
\operatorname{sp}(\Psi(\mu), M(G))=\Psi(\mu)^{\wedge}(\Gamma) \cup\{0\},
$$

for all $\mu \in \mathscr{C}$. Let $h \in \Delta M(G)$. Then $h_{\circ} \Psi$ is a complex homomorphism on $\mathscr{C}$. By Theorem 3.2(c), we see that either

$$
h \circ \Psi=0 \text { on } \mathscr{C},
$$

or there exists a $\gamma \in \Gamma$ such that

$$
h \circ \Psi(\mu)=\mu^{\wedge}(\gamma),
$$

for all $\mu \in \mathscr{C}$. In either case, $h(\Psi(\mu)) \in \mu^{\wedge}(\Gamma) \cup\{0\}$, and so by the Gelfand theory we obtain

$$
\operatorname{sp}(\Psi(\mu), M(G)) \subseteq \mu^{\wedge}(\Gamma) \cup\{0\},
$$

for all $\mu \in \mathscr{C}$. Since $\mu^{\wedge}(\Gamma) \cup\{0\}$ is a countable set, (2) and Lemma 2.6 imply (1). This concludes the proof.

We note that our corollary is analogous to Theorem 4.6.2 of [8]. Moreover, if $\alpha^{-1}(F)$ is infinite for some finite set $F \cong \Gamma$, it is not difficult to see that $\Psi$ does not even map $\mathscr{C}$ into $M_{0}(G)$ (see the proof of Theorem 4.6.2 of [8]). Thus the condition of our corollary is both necessary and sufficient to insure that $\Psi$ maps $\mathscr{C}$ into itself.

Acknowledgment. The author wishes to thank Professor J. D. Stafney for his valuable assistance in the preparation of this work.

\section{REFERENCES}

1. N. Dunford and J. Schwartz, Linear Operators, Part I, Interscience Publishers, Inc., New York, fourth printing, 1967.

2. R. E. Edwards, Fourier Series: A Modern Introduction, vol. II, Holt, Rinehart, and Winston, Inc., New York, 1967.

3. A. Figá-Talamanca and G. Gaudry, Multipliers of $L^{p}$ which vanish at infinity, J. of Functional Analysis, 7, No. 3 (1971), 475-486.

4. E. Hewitt and H. Zuckerman, Singular measures with absolutely continuous convolution squares, Proc. Cambridge Philos. Soc., 62 (1966), 399-420.

5. L. Hörmander, Estimates for translation invariant operators in $L^{p}$ spaces, Acta Math., 104 (1960), 93-140.

6. R. Larsen, The Multiplier Problem, Lecture Notes in Mathematics, Springer-Verlag, Berlin, Heidelberg, New York, 1969.

7. L. Loomis, An Introduction to Abstract Harmonic Analysis, D. Van Nostrand Company, Inc., Princeton, New Jersey, 1953.

8. W. Rudin, Fourier Analysis on Groups, Interscience tracts in pure and applied mathematics, No. 12, 2nd edition, 1967.

9. N. Varopoulos; Sets of multiplicity in locally compact abelian groups, Ann. Inst. Fourier (Grenoble), 16, Fasc. 2 (1966), 123-158.

10. - The functions that operate on $B_{0}(\Gamma)$ of a discrete group $\Gamma$, Bull. Soc. Math. France, 93 (1965), 301-321. 
11. A. Zygmund, Trigonometric Series, vol. I and II combined, Cambridge University Press, 2nd edition, 1968.

Received March 15, 1972. The results of this paper were obtained while the author held an NSF Traineeship at the University of California, Riverside.

UNIVERSITY OF CALIFORNIA, RIVERSIDE

Current Address: Institute for Advanced Study, Princeton, N. J. 


\section{PACIFIC JOURNAL OF MATHEMATICS}

\section{EDITORS}

D. Gilbarg AND J. MILGRAM

Stanford University

Stanford, California 94305

\section{R. A. Beaumont}

University of Washington

Seattle, Washington 98105

\section{J. DUGUNDJI*}

Department of Mathematics University of Southern California Los Angeles, California 90007

RICHARD ARENS

University of California Los Angeles, California 90024

\section{ASSOCIATE EDITORS}
E. F. BECKENBACH
B. H. NEUMANN
F. WOLF
K. YoshidA

\section{SUPPORTING INSTITUTIONS}

\author{
UNIVERSITY OF BRITISH COLUMBIA \\ CALIFORNIA INSTITUTE OF TECHNOLOGY \\ UNIVERSITY OF CALIFORNIA \\ MONTANA STATE UNIVERSITY \\ UNIVERSITY OF NEVADA \\ NEW MEXICO STATE UNIVERSITY \\ OREGON STATE UNIVERSITY \\ UNIVERSITY OF OREGON \\ OSAKA UNIVERSITY
}

\author{
UNIVERSITY OF SOUTHERN CALIFORNIA \\ STANFORD UNIVERSITY \\ UNIVERSITY OF TOKYO \\ UNIVERSITY OF UTAH \\ WASHINGTON STATE UNIVERSITY \\ UNIVERSITY OF WASHINGTON \\ AMERICAN MATHEMATICAL SOCIETY \\ NAVAL WEAPONS CENTER
}

The Supporting Institutions listed above contribute to the cost of publication of this Journal, but they are not owners or publishers and have no responsibility for its content or policies.

Mathematical papers intended for publication in the Pacific Journal of Mathematics should be in typed form or offset-reproduced, (not dittoed), double spaced with large margins. Underline Greek letters in red, German in green, and script in blue. The first paragraph or two must be capable of being used separately as a synopsis of the entire paper. Items of the bibliography should not be cited there unless absolutely necessary, in which case they must be identified by author and Journal, rather than by item number. Manuscripts, in duplicate if possible, may be sent to any one of the four editors. Please classify according to the scheme of Math. Rev. Index to Vol. 39. All other communications to the editors should be addressed to the managing editor, Richard Arens, University of California, Los Angeles, California, 90024.

50 reprints are provided free for each article; additional copies may be obtained at cost in multiples of 50 .

The Pacific Journal of Mathematics is issued monthly as of January 1966. Regular subscription rate: $\$ 48.00$ a year (6 Vols., 12 issues). Special rate: $\$ 24.00$ a year to individual members of supporting institutions.

Subscriptions, orders for back numbers, and changes of address should be sent to Pacific Journal of Mathematics, 103 Highland Boulevard, Berkeley, California, 94708.

PUBLISHED BY PACIFIC JOURNAL OF MATHEMATICS, A NON-PROFIT CORPORATION

Printed at Kokusai Bunken Insatsusha (International Academic Printing Co., Ltd.), 270, 3-chome Totsuka-cho, Shinjuku-ku, Tokyo 160, Japan.

* C. DePrima will replace J. Dugundji until August 1974.

Copyright (C) 1973 by

Pacific Journal of Mathematics

All Rights Reserved 


\section{Pacific Journal of Mathematics}

\section{Vol. 47, No. $2 \quad$ February, 1973}

David Parham Bellamy, Composants of Hausdorff indecomposable continua; a mapping approach ........................ 303

Colin Bennett, A Hausdorff-Young theorem for rearrangement-invariant spaces ...........................................

Roger Daniel Bleier and Paul F. Conrad, The lattice of closed ideals and $a^{*}$-extensions of an abelian l-group ...

Ronald Elroy Bruck, Jr., Nonexpansive projections on subsets of Banach

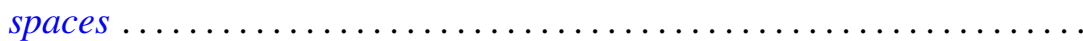

Robert C. Busby, Centralizers of twisted group algebras ............. 357

M. J. Canfell, Dimension theory in zero-set spaces ................ 393

John Dauns, One sided prime ideals ........................ 401

Charles F. Dunkl, Structure hypergroups for measure algebras . . . . . . . . . 413

Ronald Francis Gariepy, Geometric properties of Sobolev mappings ...... 427

Ralph Allen Gellar and Lavon Barry Page, A new look at some familiar spaces of intertwining operators ...........................

Dennis Michael Girard, The behavior of the norm of an automorphism of the

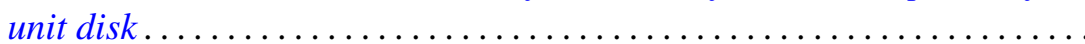

George Rudolph Gordh, Jr., Terminal subcontinua of hereditarily

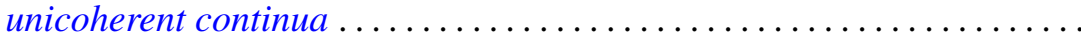

Joe Alston Guthrie, Mapping spaces and cs-networks. .

Neil Hindman, The product of $F$-spaces with $P$-spaces . 473

M. A. Labbé and John Wolfe, Isomorphic classes of the spaces $C_{\sigma}(S)$

Ernest A. Michael, On k-spaces, $k_{R}$-spaces and $k(X) \ldots$

Donald Steven Passman, Primitive group rings .

C. P. L. Rhodes, A note on primary decompositions of a pseudovaluation ...

Muril Lynn Robertson, A class of generalized functional differential equations

Ruth Silverman, Decomposition of plane convex sets. $I$.

Ernest Lester Stitzinger, On saturated formations of solvable Lie algebras................................

B. Andreas Troesch, Sloshing frequencies in a half-space by Kelvin inversion ...

L. E. Ward, Fixed point sets .

Michael John Westwater, Hilbert transforms, and a problem in scattering

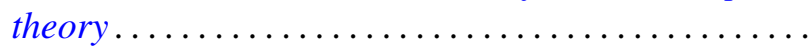

Misha Zafran, On the spectra of multipliers... 